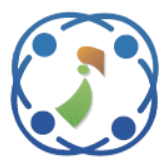

\title{
Glaucoma Detection using Bi-Dimensional Empirical Mode Decomposition from Retinal Fundus Images
}

\author{
Pooja Manghnani ${ }^{1 *}$ \\ Asmita Moghe ${ }^{1}$ \\ ${ }^{I}$ Department of Computer Science and Engineering, University Institute of Technology, RGPV Bhopal, India \\ * Corresponding author's Email: poojam2673@gmail.com
}

\begin{abstract}
Eye is a precious part of human body. But in the world, glaucoma has affected the human eye as second main cause of blindness. This disease grows very slowly in the eye and without noticing it destroyed the optic verves within the eye. Traditional glaucoma detection methods are costly and time consuming. Therefore, better methods are required to detect glaucoma more accurately. Hence, a new adaptive approach for glaucoma detection using bidimensional empirical mode decomposition (BDEMD) from retinal images is proposed. Adaptive bi-dimensional intrinsic mode functions (BDIMFs) are obtained using very simple steps, from pre-processed and coloured decomposed images, is the main idea of the proposed method. Moment and texture features contain more information hence these are extracted from decomposed BDIMFs. These features are then normalized and classified by support vector machine (SVM) with its different kernel functions. The achieved results like, accuracy, sensitivity, and specificity are $97.02 \%, 98.23 \%, 95.46 \%$, respectively for 10-fold cross validation. Experimental analysis shows that our method outperformed over the traditional methods for glaucoma detection.
\end{abstract}

Keywords: Image, Pre-processing, Bi-dimensional empirical mode decomposition, Feature extraction and normalization, Support vector machine.

\section{Introduction}

Eye is a precious part of human body. But in the world, glaucoma has affected the human eye as second main cause of blindness. This disease grows very slowly in the eye and without noticing it destroyed the optic verves within the eye [1]. The main two form of glaucoma are primary open angle glaucoma (POAG) and primary angle closure glaucoma (PACG) [2].

In the year 2013, about 64.3 million people were reported because of this eye disease and it is expected to about 111.8 million by 2040 [3]. Over all $12.3 \%$ of the eye patient reported as blindness due to glaucoma in the world [4].

There are many research papers on glaucoma detection published using public image data set. Mostly, based on pre-processing, decomposition and features extraction from retinal images with various classifiers.
Raja [5-7] proposed automated glaucoma detection approach in his three different works. His first work [5] included tri-spectrum and complex wavelets transform (CWT), second work included wavelet packet decomposition (WPD), and third work optimal hyper analytic wavelets transform (OHAWT). Raja [6] decomposed converted gray images by third level of WPD with "db3" wavelet function to explore the significance of approximation and detail coefficients. Detailed coefficient subbands images were used in the entropy and energy feature extraction process.

Gajbhiye [8] developed new method of glaucoma screening by WPD with moment feature using SVM. Ghosh [9] used new idea, in which grid colour moment features were classified by back propagation neural network (BPNN) for screening of glaucoma.

Maheshwari [10-11] proposed automated glaucoma detection approach in his two different works. His first work [10] included empirical wavelet transform (EWT) with correntropy features and 
second work included variational mode decomposition (VMD) with entropies \& fractal dimensions (FD) features. In both the works the method had been performed in 5 steps, preprocessing, decomposition, feature extraction, features normalization and selection and classification by support vector machine (SVM) for tenfold cross validation.

Araujo [12] diversity indexes in their work for glaucoma detection. They used Genetic algorithm and SVM classifier. Kirar [13] proposed automated glaucoma detection approach using discrete wavelet transform (DWT) and [14] using DWT with EWT. Kirar [15] designed an automated glaucoma detection method using compact variational mode decomposition (CVMD). Robust features were found out using post-processing, and then classified by SVM. Agrawal [16] designed an automated glaucoma detection using quasi-bivariate variational mode decomposition (QB-VMD). Robust features were found out using post-processing, and then classified by SVM. Kirar [17] designed a new glaucoma detection approach using image channels and DWT from fundus images. The obtained glaucoma detection accuracy of the proposed method was reported using SVM.

Pinto [18] proposed a new glaucoma detection approach using convolutional neural networks (CNNs). Serte [19] proposed a new glaucoma detection approach using deep learning (DL) using fundus images. Claro [20] proposed a new features extraction approach with transfer learning (TL) for glaucoma classification. Elangovan [21] proposed a new approach for glaucoma detection with CNN. Kirar [22] implemented new approach for glaucoma detection using second stage QB-VMD (SS-QBVMD) with SVM for ACRIMA database. Authors of methods [18-22] used 705 images [18].

State of the art methods are less accurate and have some limitations like; methods [5-8, 13-14, 17] are based on DWT or WPD. These are non- adaptive, limited to dyadic scale, responsible for interference and have low frequency resolution which leads nonflexible time-frequency covering. Methods [10, 14] are based on EWT, it overcomes the drawback of DWT based methods but due to having improper segmentation it leads to redundancy and interference. Methods [11, 15-16, 22] are based on VMD, it overcomes the limitations of DWT and EWT based methods, but it also suffers from boundary effects problems to perform on larger and varied data set.

Therefore, there is a requirement to increase the performance of glaucoma detection by extracting small, moderate and high frequency texture form the fundus images, which are the main discriminating characteristics of the glaucoma patient.

In this paper, a new adaptive approach for glaucoma detection using bi-dimensional empirical mode decomposition from retinal images is proposed.

The main advantage of the BDEMD is that it decomposes the fundus image in to its corresponding small, moderate and high frequency BDIMFs. BDIMFs contains pixel variations information at precise scale which helps to captures subtle pixel variations due to structural deviations in the glaucoma patient. Therefore, our main concern is to decomposes the fundus raw image in to its corresponding small, moderate and high frequency BDIMFs, so that we can easily extract various inherent most discriminating features from the raw fundus image more effectively to offer the better results.

Initially, all the images are decomposed in to different colour components. Then, BDIMFs are obtained using very simple steps, from pre-processed and colour decomposed images, it is the main idea of the proposed method. Moment and texture features contain more information hence these 17 important features are mined from decomposed BDIMFs. These features have more discriminative nature. These features are then normalized and classified using SVM with its different kernel functions. The achieved results like, accuracy, sensitivity, and specificity are reported in result section.

There are total 6 sections; remaining 5 sections are as follow: image data set is given in $2^{\text {nd }}$ section. Methodology is given in $3^{\text {rd }}$ section. Results are given in $4^{\text {th }}$ section. Section $5^{\text {th }}$ covered the discussion. Conclusion is given in $6^{\text {th }}$ section.

\section{Images data set}

Total 705 images (396 Glaucoma+ 309 Healthy) of ACRIMA public database are used [18]. The images of ACRIMA database have dimensions from $178 \times 178$ pixels to $1420 \times 1420$ and are resized to 64 $\times 64$. These are captured by Topco TRC retinal with a field of view of 35 . This project was founded by the Ministerio de Econom' 1a y Competitividad of Spain. Sample images of Glaucoma (Im685_g_ACRIMA.jpeg) and healthy (Im123_ACRIMA.jpeg) are given in Fig. 1. These images have been taken from [18].

\section{Proposed methodology}

The steps of proposed methodology are given in Fig. 2. It includes input images, resizing of images, conversion of colour image into Red, Green, Blue and Gray images, equalization and filtering of images, 


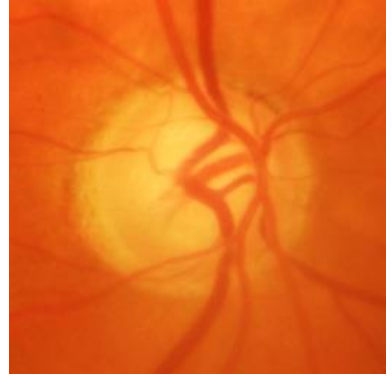

(a)

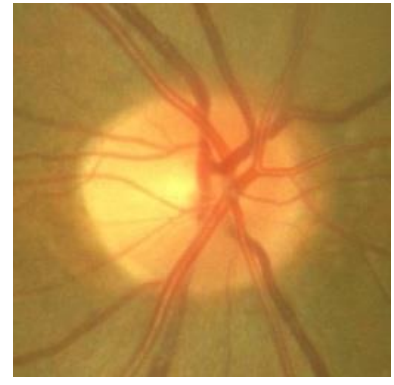

(b)
Figure. 1 Images: (a) glaucoma and (b) healthy

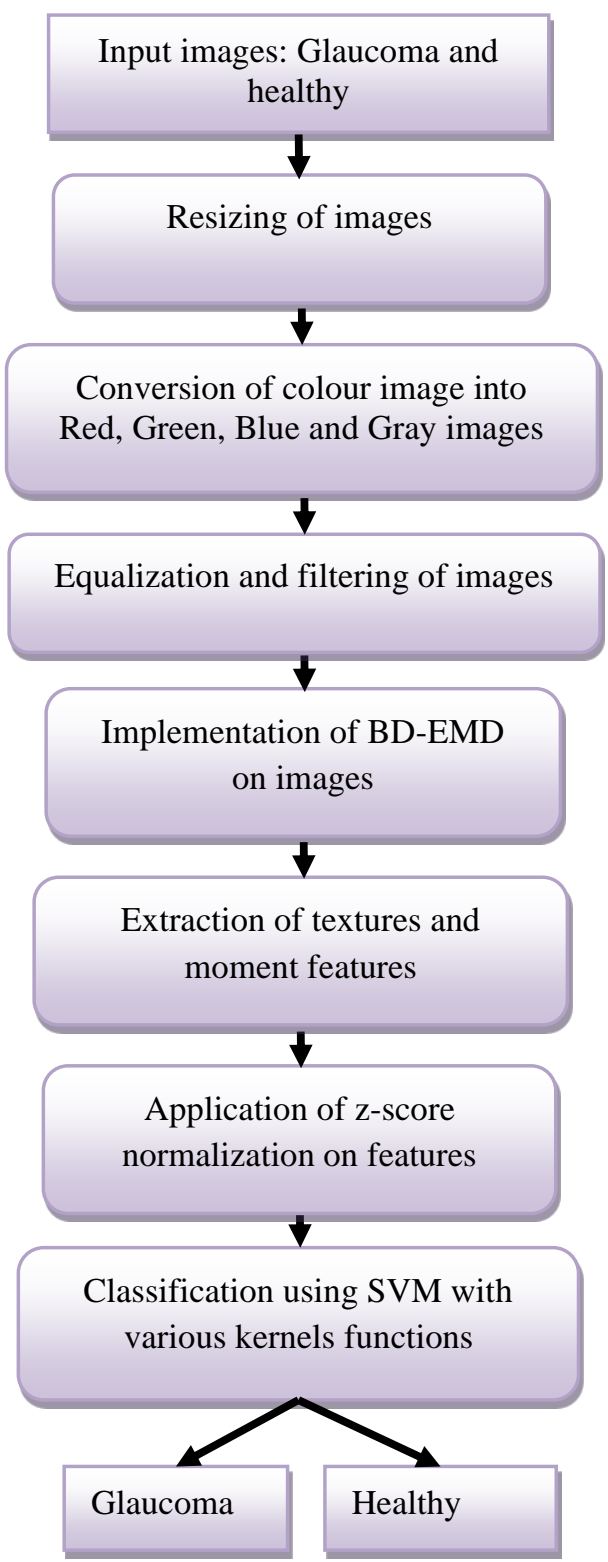

Figure. 2 Procedure of proposed methodology

implementation of bi dimensional empirical mode decomposition (BDEMD) on images, extraction of textures and moment features from decomposed BDIMFss, application of z-score normalization on features, classification using SVM with various kernels functions.

\subsection{Pre-processing}

Pre-processing is performed on images to increased image quality [1]. It improves the image quality [23]. It comprises resizing of images, conversion of colour image into Red, Green, Blue and Gray images (shown in Fig. 3), and equalization [24] followed by filtering (shown in Fig. 4).
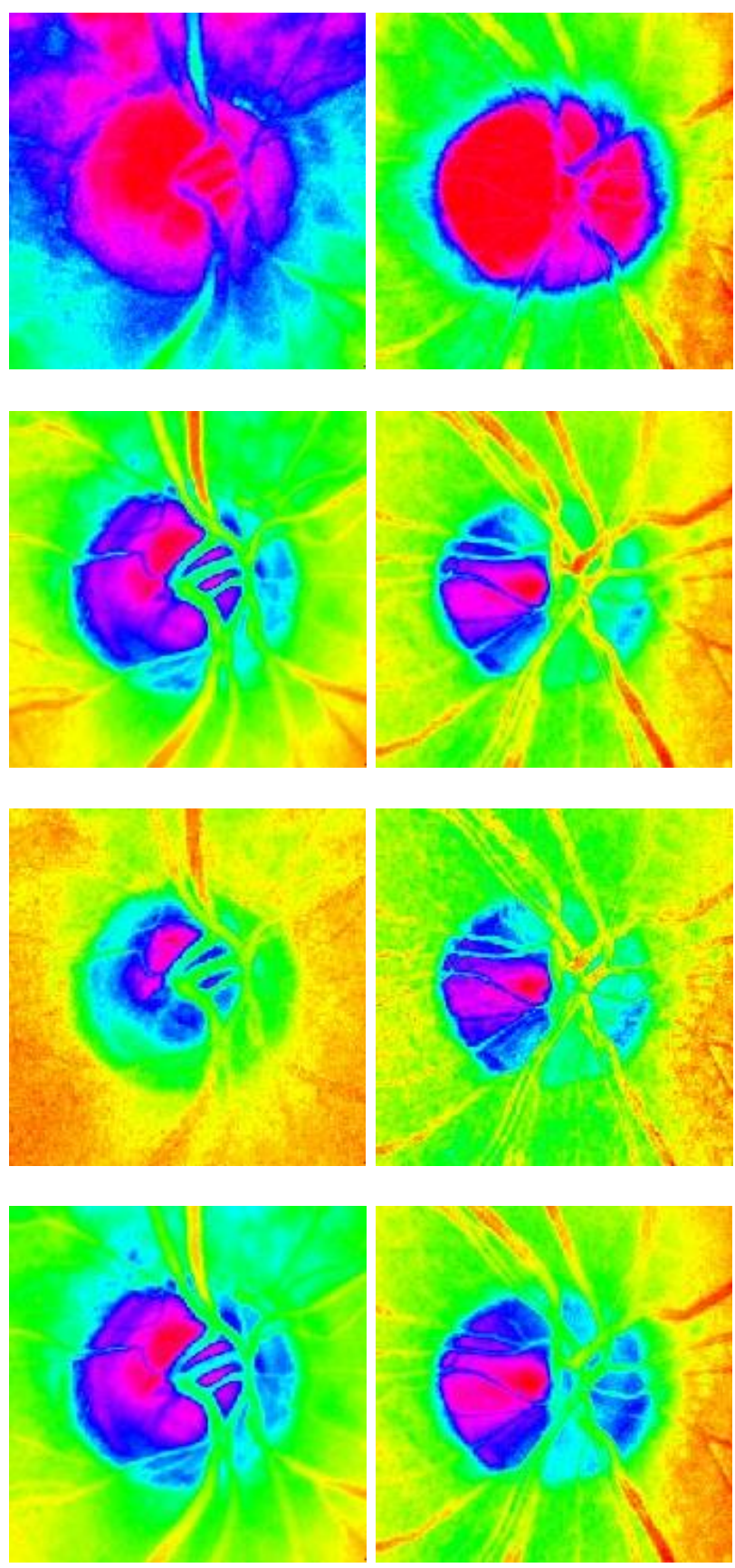

Figure. 3 Red, green blue and gray converted image: for glaucoma in $1^{\text {st }}$ column and for healthy in $2^{\text {nd }}$ column 

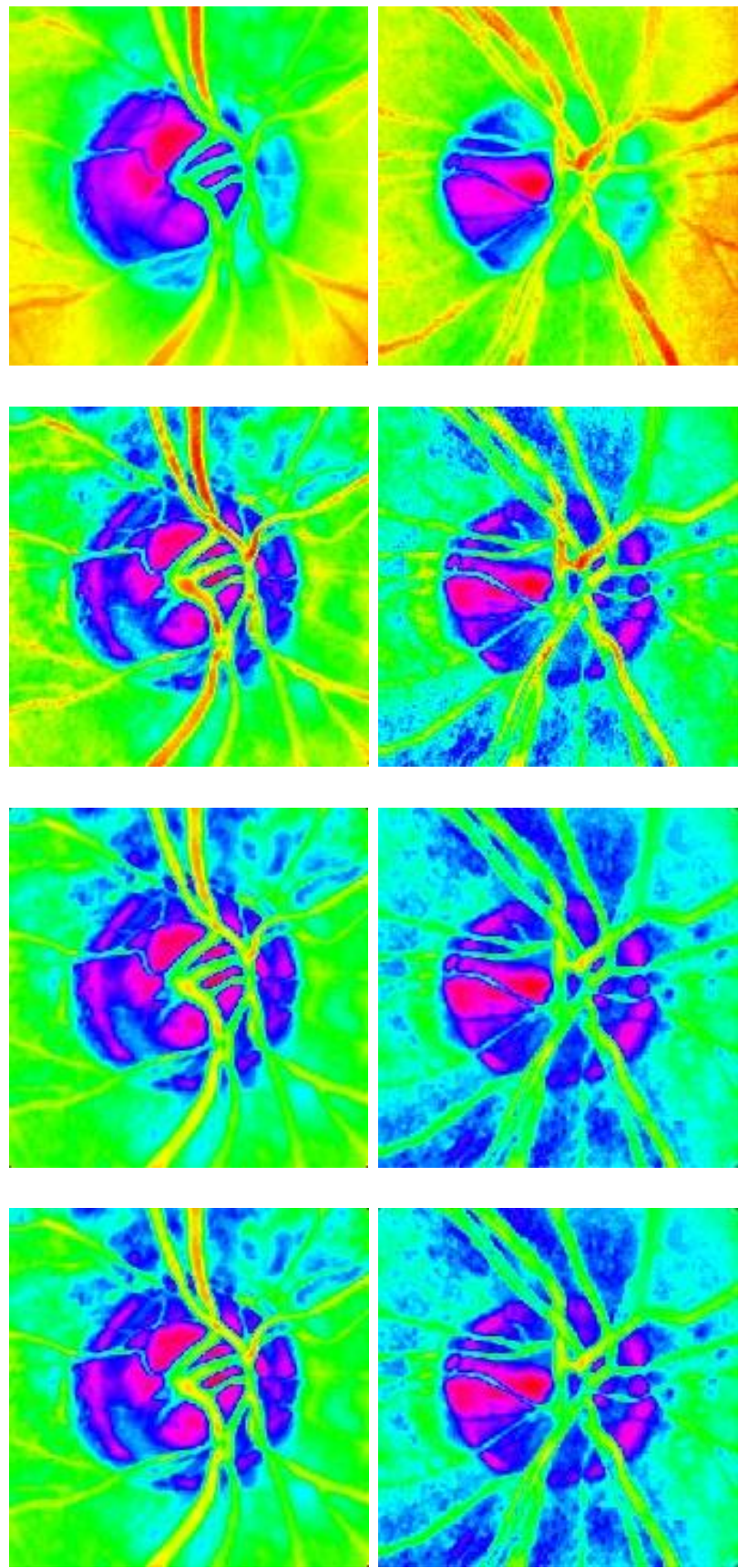

Figure.4 Green, equalized, filtered and pre-processed images: for glaucoma in $1^{\text {st }}$ column and for healthy in $2^{\text {nd }}$ column

\subsection{Bi dimensional empirical mode decomposition}

Empirical mode decomposition is abbreviated as EMD and developed by Huang. It is an adaptive in nature and used to decompose a signal, which is nonstationary and non-linear in time domain. It is independent to linearity and stationary property of signal.

The main advantage of the BDEMD is that it decomposes the fundus image in to its corresponding small, moderate and high frequency BDIMFs. BDIMFs contains pixel variations information at precise scale which helps to captures subtle pixel variations due to structural deviations in the glaucoma patient.

Huang's shifting algorithm is used to extract $\operatorname{IMF} p(z)$ from a signal $F(z)$ is given as [25-27]:

$$
F(z)=\sum_{p=1}^{P} I_{p}(z)+R_{P}(z)
$$

Where $I_{p}(z)$ is the IMFs for $p=1 \ldots, P$ and $R_{p}$ is the residue.

Nunes [25] extended EMD to Bi-dimensional EMD (BDEMD), its algorithm is summarized in the following steps is as follows [26-27]:

Step1: Calculate the maxima and minima the input image $I(x, y)$.

Step2: Upper and lower envelops are obtained using interpolation method.

Step3: Calculate mean $m$ by taking average of upper and lower envelopes.

Step4: Calculate BDIMF $R=I-m$. And check for $R$. If it is BDIMF, then move to step5 else repeat step1 to step3 using the proto-BDIMF $R$ is an input, and awaiting the newest proto-BDIMF turns to be BDIMF.

Standard deviation (SD) criterion for image is given as follow:

$$
S D C=\sum_{x=1}^{M} \sum_{y=1}^{N} \frac{\left[R_{p}(x, y)-R_{p-1}(x, y)\right]^{2}}{R_{p-1}^{2}(x, y)}
$$

If the $\mathrm{SD}$ is more than the threshold $\varepsilon$, then repeat step1 to step4 with input $R_{p}(x, y)$, otherwise $R_{p}(x, y)$ is a BDIMF $D_{p}(x, y)$.

Step5: Now, take proto-BDIMF $R$ as input for steps 1 to step 4 to calculate the remaining BDIMF.

Image is decomposed using BDEMD is given as follows:

$$
I(x, y)=\sum_{p=1}^{P} D_{p}(x, y)+R(x, y)
$$

Where $D_{p}(x, y)$ is a BDIMF and $R_{P}(x, y)$ is a residue. The BDEMD decomposes the input image as a sum of BDIMFs and residue. $D_{1}$ is the highest frequency of $I, D_{2}$ is the moderate frequency of $I$, and $R_{p}$ represents the lowest frequency of $I$.

The decomposed BDIMFs for glaucoma and healthy images are shown in Fig. 5. The BDIMFs for glaucoma in $1^{\text {st }}$ column and for healthy in $2^{\text {nd }}$ column.

\subsection{Feature extraction and normalization}

Total 17 features, 7 moment invariance and 10 texture features have been extracted from BDIMFs. These features have more discriminative nature. 
Textures features (6-chip histogram and 4-GLCM). 6-chip histogram features like kurtosis, entropy, energy, mean, variance, and skewness. 4-GLCM features are like energy, homogeneity, contrast, and correlation [13-14, 28]. In this way 17 features have been extracted from each of 4 colour components, hence over all 68 features have been extracted from one input image.

All the extracted features are normalized to improve performance of the algorithms. This research paper used z-score normalization method. It is expressed using Eq. (4) [10].
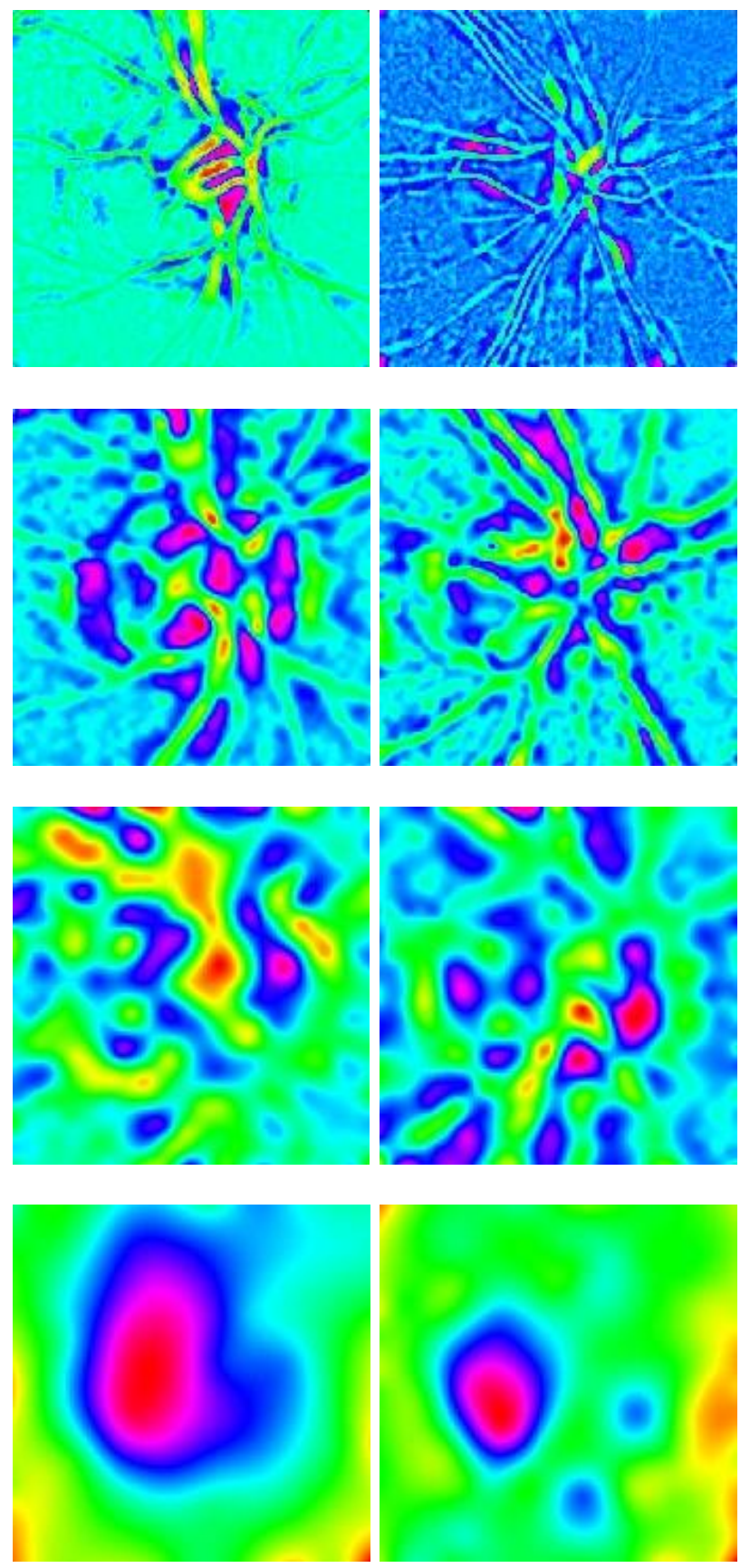

Figure. 5 BDIMFs for green image: for glaucoma in $1^{\text {st }}$ column and for healthy in $2^{\text {nd }}$ column

$$
\hat{S}=\frac{S-m(S)}{s t d(S)}
$$

Where, std=standard deviation, $\mathrm{m}=$ mean, $S=$ extracted features data.

\subsection{Support vector machine}

Support vector machine (SVM) is widely used supervised machine learning technique [13, 29].

The proposed method used SVM with different kernel function like, Linear (L), Quadratic (Q), Cubic (C), Fine Gaussian (FG), Medium Gaussian (MG), and Coarse Gaussian (CG). Therefore these are named as L-SVM), Q-SVM, C-SVM, FG-SVM, MG-SVM, and CG-SVM.

\subsubsection{Performance measures}

The following performance measures have been calculated: accuracy $(a c c)$, sensitivity ( $\operatorname{sen}$ ), and specificity (spe) using Eqs. (5-7) [14, 30].

$$
\begin{gathered}
a c c=\frac{T P+T N}{T P+T N+F P+F N} \times 100 \\
s e n=\frac{T P}{T P+F N} \times 100 \\
\text { spe }=\frac{T N}{T N+F P} \times 100
\end{gathered}
$$

Where, $\mathrm{TP}=$ TruePositive, $\mathrm{TN}=$ TrueNegative, $\mathrm{FP}=\mathrm{FalsePositive}$ and $\mathrm{FN}=$ FalseNegative .

\section{Results}

In this paper, a new adaptive approach for glaucoma detection using bi-dimensional empirical mode decomposition from retinal images is proposed. We have implemented it on 705 images (396 glaucoma and 309 healthy) of ACRIMA public image dataset [18]. Therefore features set of $705 \times 68$ is formed normalized and classified by SVM.

A normalized data is fed to L-SVM, Q-SVM, CSVM, FG-SVM, MG-SVM, and CG-SVM.

Table 1. Performance of proposed method for 3FCV

\begin{tabular}{|l|l|l|l|}
\hline Classifiers & Acc $(\boldsymbol{\%})$ & $\operatorname{Sen}(\boldsymbol{\%})$ & Spe $(\%)$ \\
\hline L-SVM & 95.04 & 96.21 & 93.53 \\
\hline Q-SVM & 96.31 & 97.47 & 94.82 \\
\hline C-SVM & 96.88 & 97.98 & 95.47 \\
\hline FG-SVM & 86.24 & 99.49 & 69.26 \\
\hline MG-SVM & 95.60 & 97.98 & 92.56 \\
\hline CG-SVM & 91.77 & 93.43 & 89.64 \\
\hline
\end{tabular}


Table 2. Performance of proposed method for $5 \mathrm{FCV}$

\begin{tabular}{|l|l|l|l|}
\hline Classifiers & $\operatorname{Acc}(\boldsymbol{\%})$ & $\operatorname{Sen}(\boldsymbol{\%})$ & $\operatorname{Spe}(\boldsymbol{\%})$ \\
\hline L-SVM & 95.04 & 95.71 & 94.17 \\
\hline Q-SVM & 96.88 & 97.98 & 95.47 \\
\hline C-SVM & 96.45 & 97.73 & 94.82 \\
\hline FG-SVM & 87.38 & 99.75 & 71.52 \\
\hline MG-SVM & 96.17 & 97.47 & 94.50 \\
\hline CG-SVM & 91.49 & 93.69 & 88.67 \\
\hline
\end{tabular}

Table 3. Performance of proposed method for $10 \mathrm{FCV}$

\begin{tabular}{|l|l|l|l|}
\hline Classifiers & $\operatorname{Acc}(\%)$ & $\operatorname{Sen}(\%)$ & Spe(\%) \\
\hline L-SVM & 95.46 & 95.45 & 95.47 \\
\hline Q-SVM & 97.02 & 98.23 & 95.47 \\
\hline C-SVM & 96.60 & 97.73 & 95.15 \\
\hline FG-SVM & 87.80 & 99.49 & 72.82 \\
\hline MG-SVM & 96.60 & 98.23 & 94.50 \\
\hline CG-SVM & 92.62 & 95.45 & 89.00 \\
\hline
\end{tabular}

We have listed the performances of our method in Tables 1-3 for 3, 5, and 10 fold cross validation, respectively. The obtained better results like acc, sen, spe, and area under curve (AUC) for the receiver operating characteristic (ROC) are $97.02 \%, 98.23 \%$, $95.47 \%$, and 0.99 , respectively for tenfold cross validation using Q-SVM.

The Fig. 6 has been plotted for accuracy versus SVM with various kernel functions for 3, 5, and 10 tenfold cross validation. In Fig. 6, Q-SVM, C-SVM, and MG-SVM reported better results for 3, 5, and 10 tenfold cross validation. But Q-SVM showed best accuracy among all with better sensitivity and specificity using tenfold cross validation. We have selected tenfold cross validation because it yielded better accuracy.

The obtained performance of the proposed method in term of ROC is also plotted in Fig. 7 for Q-SVM using tenfold cross validation. The AUC for the ROC is shown in Fig. 7.

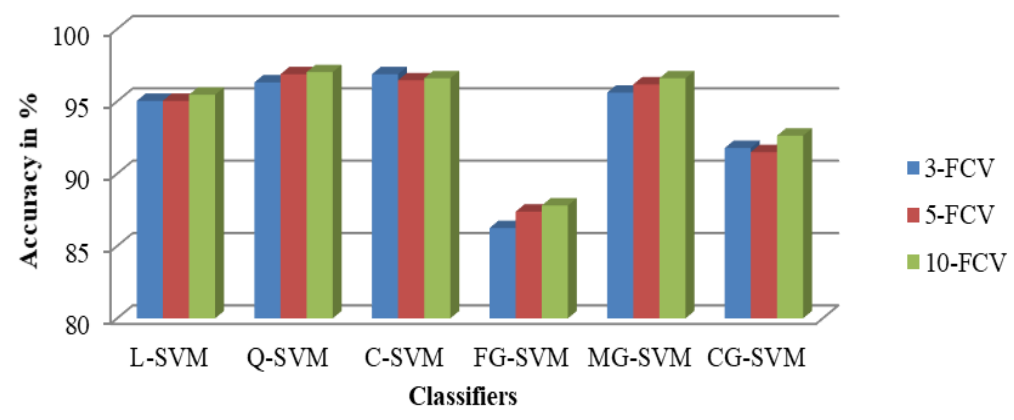

Figure. 6 Plot for accuracy versus SVM with various kernel functions for 3, 5, and 10 tenfold cross validation

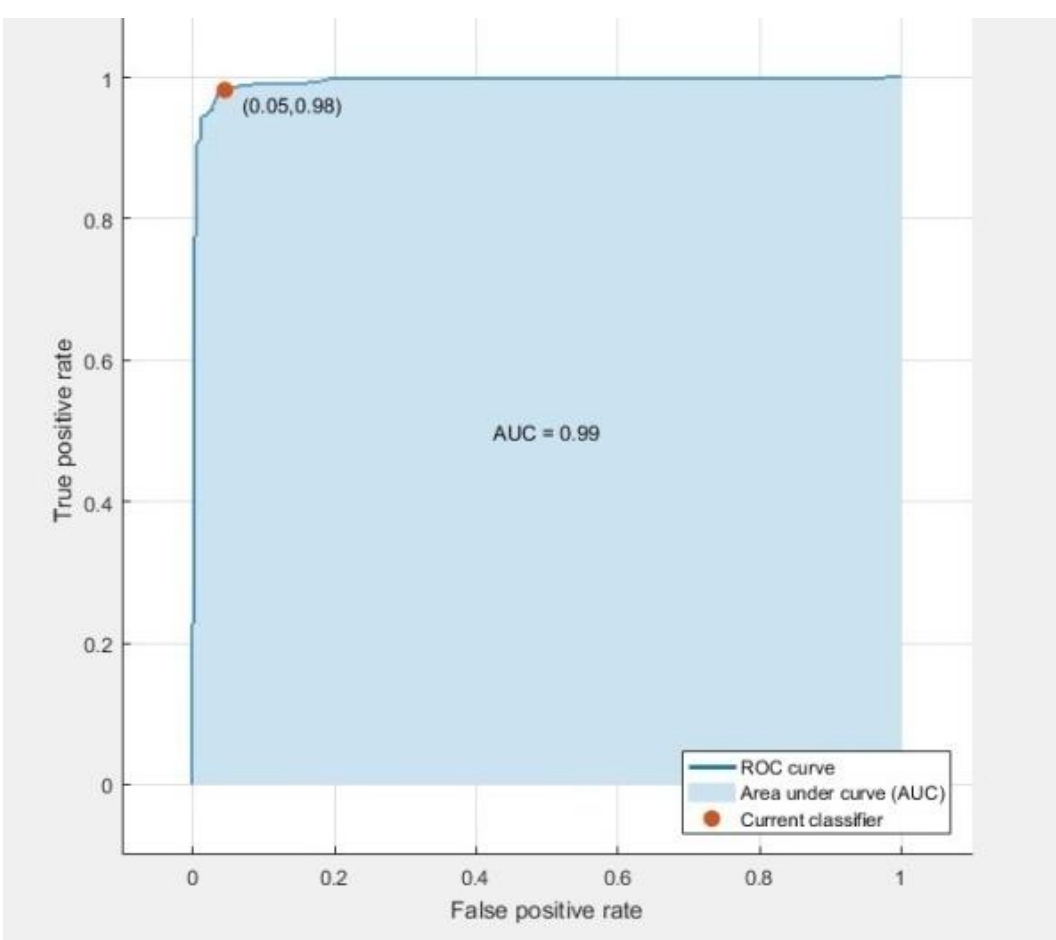

Figure. 7 ROC plot using Q-SVM for tenfold cross validation 
Table 4. Comparison of methods (in \%) for MIAG and ACRIMA image data set

\begin{tabular}{|c|c|c|c|c|c|}
\hline Year \& ref. & Images used & Classifiers & ACC & SEN & SPE \\
\hline $2017 /[10]$ & $505(250$ Glaucoma+ 255 Healthy) & SVM & 80.63 & 76 & 84.25 \\
\hline $2017 /[11]$ & $505(250$ Glaucoma+ 255 Healthy) & SVM & 81.22 & NR & NR \\
\hline $2019 /[14]$ & $505(250$ Glaucoma+ 255 Healthy) & SVM & 83.57 & 86.4 & 80.8 \\
\hline $2019 /[16]$ & $505(250$ Glaucoma+ 255 Healthy) & SVM & 86.13 & 84.8 & 97.43 \\
\hline $2020 /[17]$ & $505(250$ Glaucoma+ 255 Healthy) & SVM & 84.95 & 86 & 83.85 \\
\hline $2019 /[18]$ & $705(396$ Glaucoma+ 309 Healthy) & CNN & 70.21 & 68.93 & 70.2 \\
\hline $2019 /[19]$ & $705(396$ Glaucoma+ 309 Healthy) & GoogleNet & 65 & NR & 87 \\
\hline $2019 /[20]$ & $705(396$ Glaucoma+ 309 Healthy) & CNN & 95.31 & NR & NR \\
\hline $2020 /[21]$ & 705 (396 Glaucoma+ 309 Healthy) & CNN & 96.64 & 96.07 & 97.39 \\
\hline $2021 /[22]$ & $705(396$ Glaucoma+ 309 Healthy) & SVM & 92.06 & 91.42 & 92.89 \\
\hline Our Method & $705(396$ Glaucoma+ 309 Healthy) & SVM & 97.02 & 98.23 & 95.46 \\
\hline
\end{tabular}

\section{Discussion}

A new adaptive and computer based approach for glaucoma detection using bi-dimensional empirical mode decomposition from retinal fundus images using SVM with different kernel functions has been presented.

Raja [5-7] proposed automated glaucoma detection approach using [5] CWT, [6] WPD, and [7] OHAWT). Methods [5-7] reported an accuracy of $81 \%, 85 \%$, and $85 \%$ using ANN, ANN and SVM, respectively. Gajbhiye [8] reported an accuracy of $86.57 \%$ using WPD \& SVM. Ghosh [9] report an accuracy of $87.47 \%$ using BPNN.

Maheshwari [10, 11] used EWT and VMD and reported an accuracy of $80.7 \%$ and $81.22 \%$ using SVM, respectively from Medical Image Analysis Group (MIAG) dataset. Araujo [12] developed genetic algorithm and SVM classifier to report an accuracy of $86.37 \%$.

Kirar [13] proposed DWT and [14] DWT and EWT and reported an accuracy of $88.3 \%$ and $83.57 \%$ using SVM, respectively. Kirar [15] proposed CVMD in new work and reported an accuracy of 89.18.3\% using SVM. Agrawal [16] designed an automated glaucoma detection method using QBVMD. They reported an accuracy of $86.13 .3 \%$ using SVM. Kirar [17] designed a new glaucoma detection approach using image channels and DWT from fundus images. They reported an accuracy of $84.95 \%$ using SVM. Authors of methods $[10,11,14,16,17]$ used 505 images (250 Glaucoma +255 Healthy) from MIAG image data set by Fumero [31]. It is publicly available at http://medimrg.webs.ull.es/.

Pinto [18] proposed CNNs. The reported accuracy was 70.21\% using ACRIM image data set.
Serte [19] DL based approach and reported an accuracy of $65 \%$ using ACRIM image data set. Claro [20] proposed a new features extraction approach with TL for glaucoma classification. They reported an accuracy of $95.31 \%$ using ACRIM image data set. Elangovan [21] proposed a new approach for glaucoma detection with CNN. The reported accuracy, sensitivity, specificity were $96.64 \%$, $96.07 \%$, and $97.39 \%$, respectively, for ACRIMA database. Kirar [22] proposed a new approach for glaucoma detection using SS-QB-VMD with SVM. The reported accuracy, sensitivity, specificity was $92.06 \%, 91.42 \%$, and $92.89 \%$, respectively, for ACRIMA database [18].

Above discussed state of the art methods is less accurate and have some limitations like; methods [5$8,13,14,17]$ are based on DWT or WPD and these are non- adaptive, which also responsible for interference in the components. Methods [10,14] are based on EWT, having improper segmentation which leads to redundancy and interference. Methods [11, $15,16,22]$ are based on VMD, it overcomes the limitations of DWT and EWT based methods, but it also suffers from boundary effects problems to perform on larger and varied data set.

However, for similar dataset [18], in this paper new adaptive approach, bi-dimensional empirical mode decomposition has been implemented on preprocessed coloured components to obtain BDIMFs and extracted textures and moment features have been classified using SVM with its different kernel functions. The achieved results like, acc, sen, and spe are $97.02 \%, 98.23 \%, 95.46 \%$, respectively for 10 fold cross validation.

Furthermore, the comparison of other methods has been listed in Table 4 . Thus, Table 4 clearly 
shows that the proposed method is superior to the state of art methods because it has some benefits over the existing methods. The benefits of the proposed method are:

1. It decomposes the pre-processed fundus image in to its corresponding small, moderate and high frequency BDIMFs.

2. BDIMFs easily extract various inherent most discriminating features from the raw fundus image.

3. It is efficient to capture subtle variation in pixels.

4. It is more effective to offer the better results.

5. Reported highest accuracy of $97.02 \%$ (compared to existing methods Table 4).

6. Used 10-fold cross validation for robust system.

\section{Conclusion}

This paper presented a new adaptive and computer based approach for glaucoma detection. The coloured image is converted in to its different colour components, like red, green, blue and gray image. These are then pre-processed and decomposed using BDEMD to get BDIMFs. The main advantage of the BDEMD is that it decomposes the fundus image in to its corresponding small, moderate and high frequency BDIMFs, further these BDIMFs easily extract various inherent most discriminating features from the pre-processed fundus image. Extracted features are normalized and classified using SVM with different kernel functions. The accuracy of our method is better for tenfold cross validation using Q-SVM. The obtained acc, sen, and spe are $97.02 \%, 98.23 \%, 95.46 \%$, respectively for 10 -fold cross validation.

Furthermore, the comparison of other methods has been listed in Table 4. Thus, Table 4 and Fig. 7 clearly show that the proposed method is superior to the state of art methods.

Our method is more effective and robust to enhance the glaucoma detection performance. In future, it can be implemented on various disorders like, fatty liver, diabetes and retinopathy.

\section{Acknowledgments}

Authors want to thank to ACRIMA [18] for providing fundus images.

\section{Conflicts of Interest}

The authors declare no conflict of interest.

\section{Author contributions}

Conceptualization, methodology, data curation, formal analysis, writing-original draft preparation, $\mathrm{P}$. Manghnani; visualization, supervision, validation, writing-review and editing, A. Moghe; all authors read and approved the final manuscript.

\section{References}

[1] R. Bock, J. Meier, L. G. Ny'ul, J. Hornegger, and G. Michelson, "Glaucoma Risk Index: Automated Glaucoma Detection from Color Fundus Images", Medical Image Analysis, Vol. 14, No. 3, pp. 471-481, 2010.

[2] https://www.glaucoma.org/glaucoma/types-of-g laucoma.php (accessed on 2 Nov 2020).

[3] Y. C. Tham, X. Li, T. Y. Wong, H. A. Quigley, T. Aung, and C. Y. Cheng, "Global Prevalence of Glaucoma and Projections of Glaucoma Burden Through 2040: A Systematic Review and Meta-Analysis", Ophthalmology, Vol. 121, No. 11, pp. 2081-2090, 2014.

[4] S. Resnikoff, D. Pascolini, D. Etya'ale, I. Kocur, R. Pararajasegaram, G. P. Pokharel, and S. P. Mariotti, "Global Data on Visual Impairment in The Year 2002", Bulletin of the World Health Organization, Vol. 82, No. 11, 2004.

[5] C. Raja and N. Gangatharan, "Glaucoma Detection in Fundal Retinal Images using Trispectrum and Complex Wavelet Based Features", European Journal of Scientific Research, Vol. 97, No. 1, pp. 159-171, 2013.

[6] C. Raja and N. Gangatharan, "Appropriate SubBand Selection in Wavelet Packet Decomposition for Automated Glaucoma Diagnoses", International Journal of Automation and Computing, Vol. 12, No. 4, pp. 393-401, 2015.

[7] C. Raja and N. Gangatharan, "Optimal Hyper Analytic Wavelet Transform for Glaucoma Detection in Fundal Retinal Images", $J$ Electr Eng Technol., Vol. 10, No. 4, pp. 1899-1909, 2015.

[8] G. O. Gajbhiye and A. N. Kamthane, "Automatic Classification of Glaucomatous Images Using Wavelet and Moment Feature, In: Proc. of Annual IEEE India Conference, New Delhi, pp. 1-5, 2015.

[9] A. Ghosh, A. Sarkar, A. S. Ashour, D. BalasTimar, N. Dey, and V. E. Balas, "Grid Color Mo-Ment Features in Glaucoma Classification", International Journal of Advanced Computer Science and Applications, Vol. 6, No. 9, 2015.

[10] S. Maheshwari, R. B. Pachori, and U. R. Acharya, "Automated Diagnosis of Glaucoma 
using Empirical Wavelet Transform and Correntropy Features Extracted from Fundus Images", IEEE J. Biomed. Health Inf., Vol. 21, No. 3, pp. 903-813, 2017.

[11] S. Maheshwari, R. B. Pachori, V. Kanhangad, S. V. Bhandary, and U. R. Acharya, "Iterative Variational Mode Decomposition Based Automated Detection of Glaucoma using Fundus Images", Comput Biol Med., Vol. 88, pp. 142-149, 2017.

[12] J. D. L. Araujo, J. C. Souza, O. P. S. Neto, J. A. D. Sousa, J. D. S. D. Almeida, A. C. D. Paiva, A. C. E. Silva, G. B. Junior, and M. Gattass, "Glaucoma Diagnosis in Fundus Eye Images using Diversity Indexes", Multimed Tools Appl, 2018.

[13] B. S. Kirar and D. K. Agrawal, "Glaucoma Diagnosis using Discrete Wavelet Transform and Histogram Features from Fundus Image", International Journal of Engineering \& Technology, Vol. 7, No. 4, pp. 2546-2551, 2018.

[14] B. S. Kirar and D. K. Agrawal, "Computer Aided Diagnosis of Glaucoma Using Discrete and Empirical Wavelet Transform from Fundus Images, IET Image Processing, Vol. 13, pp. 7382, 2019.

[15] B. S. Kirar and D. K. Agrawal, "Current Research on Glaucoma Detection Using Compact Variational Mode Decomposition from Fundus Images", International Journal of Intelligent Engineering and Systems, Vol. 12, No. 3, pp. 1-10, 2019.

[16] D. K. Agrawal, B. S. Kirar, and R. B. Pachori, "Automated Glaucoma Detection Using QuasiBivariate Variational Mode Decomposition from Fundus Images", IET Image Processing Vol. 13, pp. 2401-2408, 2019.

[17] B. S. Kirar, D. K. Agrawal, and S. Kirar "Glaucoma Detection Using Image Channels and Discrete Wavelet Transform," IETE J. Research.

[18] A. D. Pinto, S. Morales, V. Naranjo, T. Kohler, J. Mossi, and A. Navea, "CNNs for Automatic Glaucoma Assessment Using Fundus Images: An Extensive Validation", Biomed Eng Online, Vol. 18, No. 29, pp. 1-19, 2019.

[19] S. Serte and A. Serener, "A Generalized Deep Learning Model for Glaucoma Detection", In: Proc. of 3rd International Symposium on Multidisciplinary Studies and Innovative Technologies, Ankara, Turkey, pp. 1-5, 2019.

[20] M. Claro, R. Veras, A. Santana, F. Araújo, R. Silva, J. Almeida, and D. Leite, "An Hybrid Feature Space from Texture Information and Transfer Learning for Glaucoma Classification",
Journal of Visual Communication and Image Representation, Vol. 64, 2019.

[21] P. Elangovan and M. K. Nath, "Glaucoma assessment from color fundus images using convolutional neural network", Int J Imaging Syst Technol, pp. 1-17, 2020.

[22] B. S. Kirar, R. S. R. Gosula, and D. K. Agrawal, "Glaucoma Detection Using SS-QB-VMD Based Fine Sub Band Images from Fundus Images," IETE Journal of Research, 2021.

[23] A. Manjaramkar and M. Kokare, "Connected Component Clustering Based Hemorrhage Detection in Color Fundus Images", International Journal of Intelligent Engineering and Systems, Vol. 11, No. 2, pp. 143-151, 2018.

[24] K. Zuiderveld, Contrast Limited Adaptive Histograph Equalization, Graphic Gems IV. San Diego, Academic Press Professional, pp. 474485, 1994.

[25] J. C. Nunes, S. Guyot, and E. Delechelle, "Texture Analysis Based on Local Analysis of The Bidimensional Empirical Mode Decomposition", Mach. Vis. Appl, Vol. 16, No. 3, pp. 177-188, 2005.

[26] P. Jianjia and T. Y. Yan, "A Mean Approximation Based Bidimensional Empirical Mode Decomposition with Application to Image Fusion," Digital Signal Processing, Vol. 50, pp. 61-71, 2016.

[27] B. S. Kirar, and D. K. Agrawal, "Comparison between empirical and variational mode decomposition based on percentage variation in entropy feature from glaucoma image," Indian Journal of Public Health Research and Development, Vol. 9, No. 9, 2018.

[28] R. C. Gonzalez and R. E. Woods, Digital Image Processing, Third edn., Pearson, UP, India, 2014.

[29] J. A. K. Suykens and J. Vandewalle, "Least Squares Support Vector Machine Classifiers", Neural Processing Letters, Vol. 9, No. 3, pp. 293-300, 1999.

[30] H. M. Premalatha, C. V. Srikrishna, "Software Fault Prediction and Classification using Cost based Random Forest in Spiral Life Cycle Model", International Journal of Intelligent Engineering and Systems, Vol. 11, No. 2, pp. 10$17,2018$.

[31] F. Fumero, S. Alayon, J. L. Sanchez, J. Sigut, and M. G. Hernandez, "RIM-ONE: An Open Retinal Image Database for Optic Nerve Evaluation," In: Proc. of 24th IEEE Int. Symposium on Computer-Based Medical Systems, Bristol, pp. 1-6, 2011. 